\title{
Clinicalfocus
}

\section{Immune reconstitution inflammatory syndrome: immune restoration disease 20 years on}

\section{Martyn A H French MD, FRACP, FRCPath, Winthrop Professor in Clinical Immunology \\ School of Pathology and Laboratory Medicine, University of Western Australia, Perth, WA. \\ martyn.french@ uwa.edu.au}

MJA 2012; 196: 318-321 doi: 10.5694/mjal2.10089
he use of antiretroviral therapy (ART) in patients with HIV infection results in reconstitution of the immune system and the restoration of immune responses against opportunistic pathogens, albeit to a variable degree. Consequently, infections by opportunistic pathogens resolve or are prevented. However, some patients experience a paradoxical worsening of treated opportunistic infections, or unmasking of a subclinical infection, during the first 3 months of ART. Based on the findings of a study undertaken over 20 years ago in patients who developed Mycobacterium avium complex (MAC) disease after commencing zidovudine monotherapy, ${ }^{1}$ followed by studies of the first patients to commence combination $\mathrm{ART}_{1}^{2,3}$ it has become clear that this is not immunodeficiency disease but immune restoration disease (IRD).

In IRD, restoration of an immune response against living or dead opportunistic pathogens causes immunopathological lesions in tissues infected by that pathogen. Many different pathogens are associated with IRD, and various terminologies have been used to describe the disease (Box 1). Immune reconstitution inflammatory syndrome (IRIS) is now most commonly used to describe this group of conditions.

The most commonly identified risk factors for IRIS are a $\mathrm{CD} 4^{+}$T-cell count of $<50 / \mu \mathrm{L}^{3,4}$ and a high pathogen load. The latter factor is exemplified by tuberculosis-associated IRIS (TBIRIS), in which disseminated TB and a high level of mycobacterial lipoarabinomannan in the urine are risk factors. 5,6

Immune reconstitution in patients with HIV may also be associated with autoimmune disease and immune-mediated inflammatory disease, such as sarcoidosis. However, the immunopathogenesis of these conditions appears to be different to that of IRIS. ${ }^{7}$

IRIS is uncommon in Australian patients with HIV because ART is usually commenced before severe immunodeficiency and opportunistic infections occur, but it may be a complication of ART in about $10 \%-40 \%$ of patients who present with low $\mathrm{CD}^{+}{ }^{+} \mathrm{T}$-cell counts. Non-tuberculous mycobacteria were the first pathogens to be associated with IRIS, ${ }^{1,4}$ but others (Box 1) are now more significant. Here, I discuss types of IRIS associated with some of these pathogens to illuminate aspects of disease pathogenesis and management.

\section{Pathogen-specific types of IRIS}

\section{Tuberculosis-associated IRIS}

The World Health Organization estimates that at least one third of the 34 million people infected with HIV worldwide are also infected with Mycobacterium tuberculosis. ${ }^{8}$ Initiation
Summary

Restoration of immune responses against opportunistic pathogens after commencing antiretroviral therapy (ART) may cause immune restoration disease (IRD) in about $10 \%-40 \%$ of HIV patients with low $\mathrm{CD}^{+} \mathrm{T}$-cell counts and usually presents clinically as a type of immune reconstitution inflammatory syndrome (IRIS).

- IRIS may be associated with many different opportunistic pathogens, but types associated with Mycobacterium tuberculosis, BCG, cryptococci, JC polyomavirus (the cause of progressive multifocal leukoencephalopathy $[P M L]$ ), hepatitis $C$ virus and hepatitis $B$ virus infection are the most informative about disease pathogenesis and management.

- $\mathrm{A} \mathrm{CD4} 4^{+}$T-cell count of $<50 / \mu \mathrm{L}$ and a high pathogen load are the most commonly identified risk factors for IRIS.

- Recovery of pathogen-specific T-cell responses and perturbations of innate immune responses before and after ART appear to cause immunopathological abnormality in tissues infected by the pathogen.

- Prevention of IRIS may be influenced by the timing of ART:

The risk of tuberculosis (TB)-associated-IRIS can be reduced by commencing ART after 8 weeks of TB treatment, but rates of AIDS or death are lower if ART is commenced during the first 4 weeks of TB treatment.

Outcomes for patients with HIV and treated cryptococcal or TB meningitis may be improved by deferring ART until the opportunistic infection is fully suppressed, but data are inadequate.

As ART is currently the only effective treatment for $\mathrm{PML}$ in patients with HIV, PML-associated IRIS cannot be prevented by manipulating the timing of ART.

- Agreater understanding of the immunopathogenesis of IRIS may lead to targeted therapies.

of ART will result in paradoxical TB-IRIS in $20 \%-25 \%$ of patients with HIV and treated TB. ${ }^{9,10}$ This presents as an exacerbation of inflammation at previous sites of disease and/or new sites of inflammation, particularly in lymph nodes. ${ }^{5}$ It has resulted in significantly increased use of health resources for patients with HIV in resource-limited countries, but mortality from TB-IRIS occurs in less than $5 \%$ of affected patients. Longer treatment of TB before ART is commenced reduces the risk of TB-IRIS,, 10 suggesting that a lower pathogen load reduces the amount of antigen that induces an immune response. 
1 Selected pathogens associated with immune restoration disease in patients with HIV during early ART

\begin{tabular}{ll} 
Pathogen & Immune restoration disease* \\
\hline $\begin{array}{l}\text { Mycobacterium tuberculosis } \\
\text { Mycobacterium avium complex } \\
\text { (MAC) }\end{array}$ & Tuberculosis-associated IRIS \\
$\begin{array}{l}\text { Mycobacterium leprae } \\
\text { BCG }\end{array}$ & Leprosy reaction associated with IRIS \\
Cryptococci & BCG-associated IRIS \\
Pneumocystis jirovecii & Cryptococcosis-associated IRIS \\
Cytomegalovirus (CMV) & Exacerbation of pneumonitis after ART \\
& CMV retinitis after ART \\
Kaposi sarcoma herpes virus & Immune recovery uveitis \\
Varicella zoster virus & Kaposi sarcoma-associated IRIS \\
& Dermatomal or multidermatomal zoster \\
Hepatitis C virus & Myelitis or Ramsay Hunt syndrome (rarely) \\
Hepatitis B virus & Hepatitis flare and/or liver enzyme level elevation after ART \\
JC polyomavirus & Hepatitis flare and/or liver enzyme level elevation after ART \\
\hline
\end{tabular}

ART = antiretroviral therapy. IRIS = immune reconstitution inflammatory syndrome. * Reflects the terminology used for, or clinical presentation of, the immune restoration disease associated with each pathogen.

Restoration of an immune response against mycobacterial antigens may also unmask $M$. tuberculosis infection that was unrecognised before ART was commenced. Consequently, higher rates of TB are observed during the first 3 months of ART in countries with high rates of endemic TB. While some patients exhibit prominent inflammation and could be considered to have "unmasking TB-IRIS", others do not and cannot be distinguished from patients who develop TB unrelated to immune reconstitution. ${ }^{5}$ Patients who develop TB during the first 3 months of ART have a 3.5 -fold increase in mortality rate compared with patients who present with $\mathrm{TB}$ at other times. ${ }^{11}$ It is therefore important to detect and commence treatment for latent or subclinical TB in patients with HIV before ART is commenced. Preliminary data suggest that detection of T cells reactive to $M$. tuberculosis antigens may be of value, ${ }^{12}$ but further studies are needed.

\section{BCG-associated IRIS}

In countries where BCG vaccine is routinely used, vaccination of children with unrecognised HIV infection may lead to subclinical BCG infection, which may then be unmasked when ART is commenced. ${ }^{13} \mathrm{Up}$ to $15 \%$ of children with HIV infection who have received BCG vaccination develop BCG-IRIS, which usually presents as regional adenitis that may suppurate and fistulate. A low proportion $(<25 \%)$ of $\mathrm{CD}^{+}$lymphocytes and a plasma HIV RNA level of $>750000$ copies/mL are risk factors for developing BCG-IRIS.

\section{Cryptococcosis-associated IRIS}

About $25 \%$ of patients with HIV and treated cryptococcal meningitis will experience cryptococcosis-associated IRIS (C-IRIS) after commencing ART. ${ }^{14}$ This usually presents as a neurological deterioration related to a "relapse" of meningitis and/or cerebral inflammation. Lymphadenitis (Box 2) and pulmonary inflammation may also occur. C-IRIS is associated with mortality in more than $25 \%$ of patients from resource-poor countries, and it is an important cause of early mortality after ART in patients with HIV from these countries. Early initiation of ART in patients with treated cryptococcal meningitis was associated with a higher rate of mortality in one study. ${ }^{15}$

\section{Progressive multifocal leukoencephalopathy- associated IRIS}

Reactivation of JC polyomavirus infection in patients with HIV infection may lead to a lytic infection of oligodendrocytes in the white matter of the brain and result in progressive multifocal leukoencephalopathy (PML). There is no established treatment for JC polyomavirus infection, so ART is the only effective therapy for PML in patients with HIV. However, about $15 \%$ of patients experience an exacerbation of PML after ART is commenced. ${ }^{16}$ Although some patients may experience a progression of the PML, most have evidence of an inflammatory response at sites of the JC polyomavirus infection, which can be demonstrated by gadolinium-enhanced magnetic resonance imaging scans of the brain or by brain biopsy. Brain biopsy usually demonstrates an inflammatory cell infiltrate, which is atypical for PML, with a predominance of $\mathrm{CD} 8^{+} \mathrm{T}$ cells.

\section{Hepatitis flare and/or liver enzyme level elevation in patients with HIV and hepatitis B or C co-infection}

Up to $25 \%$ of patients with HIV and hepatitis B virus (HBV) or hepatitis C virus (HCV) co-infection experience a flare of hepatitis and/or elevation of serum liver enzyme levels after commencing ART. ${ }^{17,18}$ Occasionally, the hepatitis may result in decompensation of liver function and, rarely, death. While drug toxicity, particularly from nevirapine, must always be considered as a cause of elevation of liver enzyme levels after commencing ART, there is emerging evidence that immune responses against HBV or HCV are also a cause. ${ }^{17,18}$ Hepatitis flares in patients with HIV and HBV co-infection are associated with a higher plasma HBV DNA level before ART, ${ }^{17}$ suggesting that pathogen load is an important determinant of disease.

\section{Immunopathogenesis of IRIS}

Given that many different types of pathogen may be associated with IRIS, and that infection by these pathogens is normally controlled by different types of immune response, it is probable that the immunopathogenesis of the different types of IRIS will differ. ${ }^{7}$

Experiments in $M$. avium-infected lymphopenic mice that have been reconstituted with $\mathrm{CD}^{+} \mathrm{T}$ cells have demonstrated that $\mathrm{CD} 4^{+} \mathrm{T}$ cells can mediate mycobacterial IRIS, at least in that experimental model. ${ }^{19}$ Similarly, mycobacterial and fungal types of IRIS in patients with HIV are associated with increased numbers of circulating pathogen-reactive $\mathrm{T}$ cells, including polyfunctional $\mathrm{CD} 4^{+} \mathrm{T}$ cells. $^{20}$ Patients with HIV and HCV co-infection who experience an increase in serum liver enzyme levels after commencing ART demonstrate higher T-cell responses to non-structural antigens of HCV in interferon- $\gamma$ enzymelinked immunosorbent spot assays, and higher serum levels of antibodies to a mixture of virus proteins, compared with patients who have no change in liver enzyme 
2 Cryptococcosis-associated immune reconstitution inflammatory syndrome presenting as necrotising granulomatous lymphadenitis

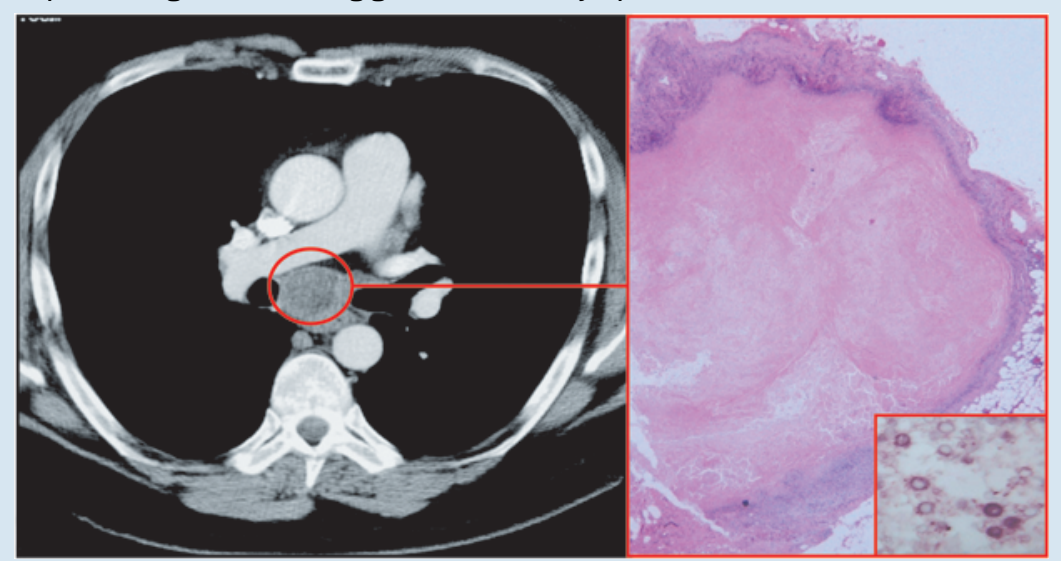

Necrotising granulomatous lymphadenitis of a subcarinal lymph node during antiretroviral therapy in a patient with HIV infection and previously treated cryptococcal meningitis. The inset shows cryptococci that were demonstrated in biopsy material but could not be cultured.

levels. ${ }^{18}$ However, an increase in pathogen-specific T cells may not be the only cause of the immunopathological abnormality, because this increase may also be observed in patients infected by the same pathogen who do not develop IRIS. ${ }^{12,21}$

Perturbations of innate immune responses may contribute to the immunopathological abnormality both before and after ART is commenced. Thus, patients who develop TB-IRIS have lower plasma levels of the chemokine CCL2 before commencing $\mathrm{ART}^{22}$ and patients who develop CIRIS have lower serum levels of tumour necrosis factor- $\alpha$, granulocyte colony-stimulating factor, granulocyte-macrophage colony-stimulating factor and vascular endothelial growth factor. ${ }^{23}$ All of these chemokines or cytokines are produced by cells of the innate immune system, particularly monocytes, and their deficiency might impair innate immune responses and lead to a higher pathogen load. After commencing ART, there is increased production of multiple pro-inflammatory chemokines and cytokines, including CXCL10 in both TB-IRIS ${ }^{22}$ and hepatitis flares in HIV-HBV co-infected patients. ${ }^{17}$ CXCL10 is a chemoattractant for $\mathrm{T}$ cells and natural killer (NK) cells.

While the immunopathogenesis of the different types of IRIS may differ depending on the pathogen, evidence is emerging that "uncoupling" of innate and adaptive immune responses against pathogens may be a common feature. ${ }^{24}$

\section{Prevention of IRIS}

As a low $\mathrm{CD}^{+} \mathrm{T}$-cell count before commencing ART is a strong predictor of IRIS, commencing ART before severe $\mathrm{CD} 4^{+} \mathrm{T}$-cell depletion occurs is a means of preventing IRIS. When commencement of ART at a very low $\mathrm{CD} 4^{+} \mathrm{T}$-cell count is unavoidable, patients should be carefully screened for infection by an opportunistic pathogen, and appropriate therapy commenced if an infection is found. As active M. tuberculosis infection may occur in patients with HIV who do not have severe $\mathrm{CD} 4^{+} \mathrm{T}$-cell depletion, screening for infection should be undertaken in all patients from TBendemic regions who have HIV. Preliminary data indicate that interferon- $\gamma$ release assays may be of value, ${ }^{12}$ but further studies are needed.

There is no evidence that any particular antiretroviral drug induces IRIS more often than other drugs. However, timing of ART is an important consideration in trying to prevent IRIS in patients with HIV and an active or recently treated infection by an opportunistic pathogen. In patients with treated TB, commencement of ART during the first 4 weeks of TB treatment increases the risk of TB-IRIS by about 2.5-fold compared with commencing ART after 8 weeks of TB treatment. ${ }^{9,10}$ However, rates of AIDS or death are reduced by about threefold when ART is initiated earlier; although data from one study ${ }^{9}$ suggest that this benefit is only seen in patients who commence ART with a CD $4^{+}$T-cell count of $<50 / \mu \mathrm{L}$.

IRIS of the central nervous system (CNS) causes more morbidity and mortality than non-CNS IRIS, ${ }^{16}$ and the highest level of attention should be paid to IRIS prevention strategies in patients with CNS infections. PML of the brain should be treated with ART as soon as possible because ART is the only effective therapy for PML in patients with HIV. Outcomes for patients with HIV and treated cryptococcal or TB meningitis may be better if ART is deferred until the opportunistic infection has been fully suppressed, ${ }^{5,15}$ but this is an area of uncertainty and further studies are needed.

HBV infection can be treated at the same time as HIV infection using the antiretroviral drugs tenofovir and lamivudine or emtricitabine but, nevertheless, about $20 \%$ of patients experience hepatitis flares. ${ }^{17}$ Flares of hepatitis and/or elevation of liver enzyme levels in patients with HIV and HCV co-infection might be avoided by treating the HCV infection before the HIV infection. However, the long-term effects of ART-associated hepatitis flares are unclear, and early introduction of ART may be beneficial overall through a reduction in the rate of liver fibrosis. Opinion is divided on the optimal timing of ART in patients with HIV and HCV co-infection, and randomised controlled trials are needed. ${ }^{25}$

\section{Management of IRIS}

Corticosteroid therapy is effective in patients who experience severe TB-IRIS. ${ }^{26}$ Corticosteroid or other anti-inflammatory therapies may also be effective for treating other types of IRIS, such as PML-associated IRIS, ${ }^{16}$ but evidence-based guidelines for their use are needed. ART should only be ceased in severe IRIS, particularly when it is life-threatening.

\section{Conclusions}

During the past 20 years, IRD has evolved from an unusual presentation of MAC disease after commencing zidovudine therapy, of uncertain significance, into the various types of IRIS that affect many thousands of HIV-infected patients commencing ART around the world. Increased knowledge about the immunopathogenesis of this group of conditions should lead to improved diagnostic laboratory testing and targeted therapies. In addition to perturbations of innate and adaptive immune responses, 
pathogen load appears to influence disease pathogenesis. Future research should examine strategies for reducing pathogen load before commencing ART, as well as therapeutically modulating the immunopathological processes that cause IRIS.

Competing interests: No relevant disclosures.

Provenance: Not commissioned; externally peer reviewed.

1 French MA, Mallal SA, Dawkins RL. Zidovudine-induced restoration of cellmediated immunity to mycobacteria in immunodeficient HIV-infected patients. AIDS 1992; 6: 1293-1297.

2 John M, Flexman J, French MA. Hepatitis C virus-associated hepatitis following treatment of HIV-infected patients with HIV protease inhibitors: an immune restoration disease? AIDS 1998; 12: 2289-2293.

3 French MA, Lenzo N, John M, et al. Immune restoration disease after the treatment of immunodeficient HIV-infected patients with highly active antiretroviral therapy. HIV Med 2000; 1: 107-115.

4 Müller M, Wandel S, Colebunders R, et al. Immune reconstitution inflammatory syndrome in patients starting antiretroviral therapy for HIV infection: a systematic review and meta-analysis. Lancet Infect Dis 2010; 10: 251-261.

5 Meintjes G, Lawn SD, Scano F, et al. Tuberculosis-associated immune reconstitution inflammatory syndrome: case definitions for use in resourcelimited settings. Lancet Infect Dis 2008; 8: 516-523.

6 Conesa-Botella A, Loembé MM, Manabe YC, et al. Urinary lipoarabinomannan as predictor for the tuberculosis immune reconstitution inflammatory syndrome. J Acquir Immune Defic Syndr 2011; 58 : 463-468.

7 French MA. HIV/AIDS: immune reconstitution inflammatory syndrome: a reappraisal. Clin Infect Dis 2009; 48: 101-107.

8 World Health Organization. TB/HIV facts 2011. http://www.who.int/tb/ publications/TBHIV_Facts_for_2011.pdf (accessed Mar 2012).

9 Abdool Karim SS, Naidoo K, Grobler A, et al. Integration of antiretroviral therapy with tuberculosis treatment. NEngl J Med 2011; 365: 1492-1501.

10 Blanc FX, Sok T, Laureillard D, et al; CAMELIA (ANRS 1295-CIPRA KH001) Study Team. Earlier versus later start of antiretroviral therapy in HIV-infected adults with tuberculosis. NEngl J Med 2011; 365: 1471-1481.

11 Koenig SP, Riviere C, Leger P, et al. High mortality among patients with AIDS who received a diagnosis of tuberculosis in the first 3 months of antiretroviral therapy. Clin Infect Dis 2009; 48: 829-831.

12 Elliott JH, Vohith K, Saramony S, et al. Immunopathogenesis and diagnosis of tuberculosis and tuberculosis-associated immune reconstitution inflammatory syndrome during early antiretroviral therapy. J Infect Dis 2009; 200: 1736-1745.
13 Rabie H, Violari A, Duong T, et al. Early antiretroviral treatment reduces risk of bacille Calmette-Guérin immune reconstitution adenitis. Int J Tuberc Lung Dis 2011; 15: 1194-1200.

14 Haddow LJ, Colebunders R, Meintjes G, et al. Cryptococcal immune reconstitution inflammatory syndrome in HIV-l-infected individuals: proposed clinical case definitions. Lancet Infect Dis 2010; 10: 791-802.

15 Makadzange AT, Ndhlovu CE, Takarinda K, et al. Early versus delayed initiation of antiretroviral therapy for concurrent HIV infection and cryptococcal meningitis in sub-Saharan Africa. Clin Infect Dis 2010; 50: 1532-1538.

16 Martin-Blondel G, Delobel P, Blancher A, et al. Pathogenesis of the immune reconstitution inflammatory syndrome affecting the central nervous system in patients infected with HIV. Brain 2011; 134: 928-946.

17 Crane M, Oliver B, Matthews G, et al. Immunopathogenesis of hepatic flare in $\mathrm{HIV} /$ hepatitis B virus (HBV)-coinfected individuals after the initiation of HBVactive antiretroviral therapy. J Infect Dis 2009; 199: 974-981.

18 Cameron BA, Emerson CR, Workman C, et al. Alterations in immune function are associated with liver enzyme elevation in HIV and HCV co-infection after commencement of combination antiretroviral therapy. J Clin Immunol 2011; 31 : 1079-1083.

19 Barber DL, Mayer-Barber KD, Antonelli LR, et al. Thl-driven immune reconstitution disease in Mycobacterium avium-infected mice. Blood 2010; 116 3485-3493.

20 Mahnke YD, Greenwald JH, Dersimonian R, et al. Selective expansion of polyfunctional pathogen-specific CD4+ T cells in HIV-l-infected patients with immune reconstitution inflammatory syndrome. Blood 2012 Jan 4. [Epub ahead of print.]

21 Meintjes G, Wilkinson KA, Rangaka MX, et al. Type 1 helper T cells and FoxP3positive $T$ cells in HIV-tuberculosis-associated immune reconstitution inflammatory syndrome. Am J Respir Crit Care Med 2008; 178: 1083-1089.

22 Oliver BG, Elliott JH, Price P, et al. Mediators of innate and adaptive immune responses differentially affect immune restoration disease associated with Mycobacterium tuberculosis in HIV patients beginning antiretroviral therapy. J Infect Dis 2010; 202: 1728-1737.

23 Boulware DR, Meya DB, Bergemann TL, et al. Clinical features and serum biomarkers in HIV immune reconstitution inflammatory syndrome after cryptococcal meningitis: a prospective cohort study. PLoS Med 2010; 7: el000384.

24 Barber DL, Andrade BB, Sereti I, Sher A. Immune reconstitution inflammatory syndrome: the trouble with immunity when you had none. Nat Rev Microbiol 2012; $10: 150-156$

25 Jones M, Núñez M. HIV and hepatitis C co-infection: the role of HAART in HIV/ hepatitis C virus management. Curr Opin HIV AIDS 2011; 6: 546-552.

26 Meintjes G, Wilkinson RJ, Morroni C, et al. Randomized placebo-controlled trial of prednisone for paradoxical tuberculosis-associated immune reconstitution inflammatory syndrome. AIDS 2010; 24: 2381-2390. 